\title{
Predição da carga máxima a partir do número máximo de repetições com cargas submáximas para mulheres
}

\author{
Prediction of the one-repetition maximum based on the \\ maximum number of repetitions with submaximal loads \\ in women
}

\author{
Ursula Ferreira Julio \\ Valéria Leme Gonçalves Panissa \\ Emerson Franchini
}

1 Universidade de São Paulo. Escola de Educação Física e Esporte. Departamento de Esporte, São Paulo, SP, Brasil.

Recebido em 16/02/11 Revisado em 31/03/11 Aprovado em 05/05/11
Resumo - O objetivo do presente estudo foi determinar equações que pudessem estimar a carga correspondente à carga máxima (1RM) a partir do número máximo de repetições com cargas submáximas no supino horizontal, puxador costas, e mesa flexora em mulheres familiarizadas com treinamento de força $(\mathrm{n}=20 ; 22 \pm 3$ anos; $61 \pm 7 \mathrm{~kg} ; 165 \pm 4 \mathrm{~cm})$. $\mathrm{O}$ primeiro teste foi o teste de $1 \mathrm{RM}$. Posteriormente, as participantes realizaram o número máximo de repetições nas intensidades de 70, 80 e 90\% de 1RM em todos os exercícios, em diferentes dias. Para determinar cada equação, foi realizada a análise de regressão linear múltipla. Dois critérios foram considerados para aceitar cada equação: o coeficiente de determinação ajustado $\left(\mathrm{R}^{2}\right.$ aj. $\left.>0,80\right)$ e o erro percentual $(\mathrm{EP}<10 \%)$. Para todos os exercícios, as equações atingiram os critérios: supino horizontal: $\mathrm{R}^{2}$ aj. $=0,89 ; \mathrm{EP}=5 \%$; puxador costas: $\mathrm{R}^{2}$ aj. $=0,84 ; \mathrm{EP}=5 \%$; mesa flexora: $\mathrm{R}^{2}$ aj. $=0,82 ; \mathrm{EP}=7 \%$. Para a utilização das equações elaboradas, é necessário escolher uma carga aleatória e o número de repetições realizados nesta mesma carga para encontrar o valor de $1 \mathrm{RM}$. Recomenda-se que o número de repetições esteja dentro da variação observada neste estudo: supino horizontal (1 - 21), puxador costas $(2-20)$ e mesa flexora $(1-14)$.

Palavras-chave: Modelos lineares; Exercício; Força muscular.

Abstract - The objective of the present study was to determine equations that could be used to estimate the load corresponding to the one-repetition maximum (1RM) based on the maximum number of repetitions (MNR) using submaximal loads in bench press, lat machine pull-down and leg curl exercises in strength-trained women ( $n=20 ; 22 \pm 3$ years; $61 \pm 7 \mathrm{~kg} ; 165 \pm 4 \mathrm{~cm}$ ). The first test was the 1RM test. Next, the subjects performed the MNR test at intensities of 70, 80 and $90 \%$ of $1 R M$ for all exercises on different days. A multiple linear regression model was used for the determination of each equation. Two criteria were applied to accept the equation: adjusted $R^{2}$ value (adj. $\left.R^{2}>0.80\right)$ and percent error $(P E<10 \%)$. For all exercises, the equations met the two criteria: bench press: $a d j R^{2}=0.89 ; P E=5 \%$; lat machine pull-down: adj $R^{2}=0.84$; $P E=5 \%$; leg curl: adjR $R^{2}=0.82 ; P E=7 \%$. The application of the equations developed will require the selection of a random load and the number of repetitions performed at the same load to identify 1RM. It is recommended that the number of repetitions be within the range observed in this study: bench press (1-21), lat machine pull-down (2-20), and leg curl (1-14).

Key words: Linear models; Exercise; Muscle strength 


\section{INTRODUÇÃO}

A crescente procura pelo treinamento de força tem incentivado a busca por parâmetros eficientes que possam ser utilizados para a sua avaliação e prescrição ${ }^{1}$. O teste de uma repetição máxima (1RM) tem sido utilizado como medida de avaliação da força máxima e para prescrição do exercício ${ }^{2-4}$, pois a partir da carga máxima levantada pode-se identificar cargas relativas, determinando a intensidade do exercício e possibilitando adaptações e benefícios específicos ${ }^{5}$.

Para a aplicação desse teste, o executante realiza um aquecimento específico no exercício que será realizado o teste, seguido por várias tentativas com carga máxima até que a falha em executar o movimento corretamente seja observada. Normalmente, o valor de 1RM é encontrado entre três e seis tentativas ${ }^{6,7} \mathrm{com}$ intervalo de recuperação entre três e cinco minutos ${ }^{7,8}$. Desta forma, para um único exercício, a realização deste teste requer aproximadamente 30 minutos, o que poderia atrapalhar a rotina do treinamento ${ }^{6}$. Consequentemente, este teste é pouco utilizado em clubes e academias pela dificuldade de operacionalização, pois dependem de grande direcionamento de recursos humanos e alta demanda em termos de tempo.

Devido a esta dificuldade, a prescrição do treinamento tem sido realizada com um número pré-determinado de repetições associado com uma dada intensidade. Porém, para os diferentes grupos musculares é observada diferença no número máximo de repetições executados na mesma intensidade relativa ${ }^{2}$. Sendo assim, é importante que se elabore uma maneira de quantificar a carga máxima (1RM), sem que seja preciso aplicar o teste propriamente dito. Tentando atender essa necessidade, alguns estudos foram conduzidos para determinar equações preditivas de 1RM a partir de diferentes variáveis. Dentre estas variáveis, destacam-se as antropométricas ${ }^{9}$, o número de repetições em exercícios com cargas fixas ${ }^{7}$ e o número máximo de repetições realizado com uma carga aleatória ${ }^{10,11}$.

Muitas das equações foram desenvolvidas utilizando como amostra homens e poucas utilizaram mulheres $^{12}$. Porém, tem sido demonstrado que as mulheres podem ser mais resistentes à fadiga, principalmente em contrações submáximas ${ }^{13}$. Por isso, seria um equívoco utilizar as equações desenvolvidas com homens para as mulheres, ficando clara a necessidade da elaboração de equações específicas para o gênero feminino.

O presente estudo testou a hipótese da existência de uma relação linear entre o número máximo de repetições realizado em cargas submáximas e a força máxima (1RM). Sendo assim, teve como objetivo determinar equações que estimassem a carga correspondente a 1RM a partir do número máximo de repetições com carga fixa, para mulheres familiarizadas com o treinamento de força no supino horizontal, puxador costas e mesa flexora.

\section{PROCEDIMENTOS METODOLÓGICOS}

\section{Amostra}

Participaram deste estudo 20 mulheres saudáveis, que concordaram em participar desse estudo voluntariamente, após a leitura e assinatura do termo de consentimento informado. O referido estudo foi aprovado pelo Comitê de Ética em Pesquisa da Universidade Presbiteriana Mackenzie, processo n.584/05/04

Os critérios de inclusão foram que as participantes apresentassem idade entre 18 e 35 anos, familiarização com o treinamento de força por período superior a seis meses, e que participassem de programas típicos de academia, ou seja, sem fins competitivos. As participantes que apresentaram algum tipo de desordem ortopédica, neuromuscular e cardiovascular, ou alguma outra patologia que impedisse ou limitasse a realização de exercícios, foram excluídas do estudo.

\section{Desenho experimental}

Durante a coleta de dados, foi solicitado às participantes que mantivessem a sua dieta habitual, além de não praticarem atividade física severa nas 24 horas precedentes aos testes. $\mathrm{O}$ procedimento para a coleta de dados foi dividido em quatro sessões distintas para cada uma das participantes, separadas por, no mínimo, 48 horas de intervalo. Na primeira sessão, foram obtidos os dados de massa corporal $(\mathrm{kg})$, estatura $(\mathrm{cm})$ e idade (anos) e realizado o teste de 1RM no supino horizontal, puxador costas e mesa flexora. Com os valores obtidos, foram calculados 70, 80 e 90\% para cada exercício. Na sessão seguinte, foi determinado aleatoriamente um dos percentuais de 1RM e solicitado que a participante realizasse o maior número de repetições corretas até a exaustão voluntária em todos os exercícios. Este procedimento foi repetido em três sessões, para que a participante realizasse os três exercícios com todos os percentuais preconizados. Em todas as sessões houve encorajamento verbal para motivar as participantes.

Para um melhor controle do movimento foi definida uma padronização para os exercícios analisados, sendo esta monitorada durante os testes. A ordem de execução dos exercícios foi supino horizontal, puxador costas e mesa flexora. 
A velocidade utilizada no teste de 1RM e no teste do número máximo de repetições não foi pré-estipulada. A instrução fornecida às avaliadas foi que as mesmas executassem as repetições na velocidade que estavam acostumadas a executar durante o treinamento, e que essa fosse consistente intra e entre as sessões de teste.

\section{Teste de 1RM}

A força dinâmica máxima foi determinada mediante a carga máxima em que a participante foi capaz de executar apenas um movimento (1RM), realizados nos aparelhos e acessórios da marca Portico ${ }^{\circledR}$. Para este estudo, o incremento de carga oferecida entre as tentativas foi de $5 \%$. Os incrementos foram feitos com anilhas e halteres. Foi necessário o uso dos halteres, visto que, em alguns exercícios (puxador costas e mesa flexora) o incremento do aparelho era superior a $5 \%$. Para tanto, foram seguidas as recomendações da Sociedade Americana de Fisiologia do Exercício ${ }^{14}$. Além disso, não houve o controle do ritmo de execução das repetições no intuito de se aproximar ao máximo do modo como esses exercícios são executados em sessões típicas. $\mathrm{O}$ intervalo de descanso foi de três a cinco minutos, o número de tentativas não foi superior a cinco.

\section{Teste do Número Máximo de Repetições}

Para a realização do teste de número máximo de repetições, primeiramente, a participante executou um aquecimento geral de três a cinco minutos no cicloergômetro. Em seguida, foi realizado um aquecimento específico em cada exercício de 12 repetições a 50\% de 1RM, seguido de um intervalo de dois minutos. Posteriormente, o número máximo de repetições até exaustão voluntária com a intensidade sorteada para a sessão, não sendo permitida pausa entre a fase concêntrica e excêntrica, e entre as repetições. Além da exaustão voluntária, o teste foi finalizado quando a participante não conseguiu realizar o movimento conforme a prévia explicação do avaliador. Este procedimento foi seguido em todos os exercícios, sendo que, entre eles, foi permitido à participante intervalo de dois minutos. Nas sessões subsequentes, a participante repetiu o mesmo procedimento até que ela concluísse todos os percentuais de 1RM propostos.

\section{Análise estatística}

Para elaboração das equações de predição foi utilizada a análise de regressão linear múltipla. A variável dependente para este estudo foi a carga equivalente a 1RM em cada exercício. As variáveis independentes foram a carga e o número máximo de repetições realizados para cada um dos diferentes percentuais. As equações foram consideradas válidas se o coeficiente de determinação ajustado $\left(\mathrm{R}^{2}\right.$ aj.) fosse igual ou superior a 0,80. Além disso, o erro padrão da estimativa (EPE) gerado pela equação não deveria exceder $10 \%$ do valor médio mensurado para o grupo, representado pelo erro percentual (EP). As análises foram feitas no software Statistical Package Minitab Version 15.1.

\section{RESULTADOS}

As características gerais das participantes foram (média \pm desvio padrão): (1) massa corporal de $61 \pm 7 \mathrm{~kg}$; (2) estatura de $165 \pm 4 \mathrm{~cm}$; (3) idade de $22 \pm 3$ anos; (4) 1RM supino horizontal - $35 \pm 5 \mathrm{~kg}$; (5) $1 \mathrm{RM}$ puxador costas - $39 \pm 5 \mathrm{~kg}$; (6) $1 \mathrm{RM}$ mesa flexora $-26 \pm 4 \mathrm{~kg}$.

Na Tabela 1, é apresentado o número de repetições nos diferentes percentuais, isoladamente, e a amplitude para todos os percentuais em conjunto para cada um dos exercícios.

As equações geradas em cada exercício supino horizontal, puxador costas e mesa flexora, considerando todas as intensidades avaliadas, são apresentadas na Tabela 2. Cada equação é acompanhada do respectivo $\mathrm{R}^{2}$ aj., EPE e EP.

Tabela 1 - Número de repetições em cada percentual isoladamente, e amplitude para todos os percentuais em conjunto (70-90\%) realizados por mulheres familiarizadas com o treinamento de força $(n=20)$.

\begin{tabular}{|c|c|c|c|c|}
\hline & & Média & $\mathrm{DP}$ & Amplitude \\
\hline $70 \% 1 \mathrm{RM}$ & $\begin{array}{c}\text { Supino horizontal } \\
\text { Puxador costas } \\
\text { Mesa flexora }\end{array}$ & $\begin{array}{c}14 \\
14 \\
9\end{array}$ & $\begin{array}{l}3 \\
3 \\
3\end{array}$ & $\begin{array}{l}9-21 \\
8-20 \\
5-14\end{array}$ \\
\hline $80 \% 1 \mathrm{RM}$ & $\begin{array}{c}\text { Supino horizontal } \\
\text { Puxador costas } \\
\text { Mesa flexora }\end{array}$ & $\begin{array}{l}8 \\
8 \\
6\end{array}$ & $\begin{array}{l}3 \\
2 \\
2\end{array}$ & $\begin{array}{l}4-15 \\
4-13 \\
3-11\end{array}$ \\
\hline $90 \% 1 \mathrm{RM}$ & $\begin{array}{c}\text { Supino horizontal } \\
\text { Puxador costas } \\
\text { Mesa flexora }\end{array}$ & $\begin{array}{l}4 \\
4 \\
3\end{array}$ & $\begin{array}{l}2 \\
1 \\
1\end{array}$ & $\begin{array}{l}1-8 \\
2-7 \\
1-5\end{array}$ \\
\hline $70-90 \%$ & $\begin{array}{c}\text { Supino horizontal } \\
\text { Puxador costas } \\
\text { Mesa flexora }\end{array}$ & $\begin{array}{l}9 \\
9 \\
6\end{array}$ & $\begin{array}{l}5 \\
5 \\
3\end{array}$ & $\begin{array}{l}1-21 \\
2-20 \\
1-14\end{array}$ \\
\hline
\end{tabular}


Tabela 2 - Equações preditivas de uma repetição máxima para os exercícios supino horizontal (SH), puxador costas (PC) e mesa flexora (MF) em mulheres familiarizadas com o treinamento de força $(n=20)$.

\begin{tabular}{|c|c|c|c|c|}
\hline \multicolumn{5}{|c|}{ Desenvolvimento das equações } \\
\hline & Equação & $\mathrm{R}^{2}$ aj. & $\mathrm{EPE}(\mathrm{kg})$ & $\mathrm{EP}(\%)$ \\
\hline $\mathrm{SH}$ & $0,973+(0,520 *$ repetições $)+\left(1,057^{*}\right.$ carga $)$ & 0,89 & 1,66 & 5 \\
\hline PC & - 0,162 + (0,652*repetições $)+(1,080 *$ carga $)$ & 0,84 & 1,96 & 5 \\
\hline MF & $-1,991+\left(0,556^{*}\right.$ repetições $)+\left(1,188^{*}\right.$ carga $)$ & 0,82 & 1,79 & 7 \\
\hline
\end{tabular}

Em relação às equações geradas (Tabela 2), podemos observar que todas atingiram o ponto de corte pré-estabelecido, considerando-se o $\mathrm{R}^{2}$ aj. e o EP.

\section{DISCUSSÃO}

De acordo com os achados do presente estudo, foi possível observar que o número máximo de repetições realizado em cargas submáximas (70, 80 e 90\% de 1RM) apresenta relação com a carga de 1RM e, portanto, pode ser utilizado para predizer a carga de 1RM, assim como reportado em outros estudos ${ }^{12,15}$.

A relação entre cargas submáximas e número de repetições tem sido apontada por alguns estudos como linear ${ }^{3,16,17}$, embora outros estudos apresentem essa relação como não-linear ${ }^{10,18}$. Vale também destacar que essa relação tem sido muito estudada em homens, mas apenas uma pequena quantidade de estudos objetivou estudar essa relação em mulheres ${ }^{12}$.

As equações geradas para predizer a carga de 1RM nos exercícios supino horizontal, puxador costas e mesa flexora, considerando o número máximo de repetições realizado em cargas submáximas, apresentou bons valores da variância explicada demonstrados pelo $\mathrm{R}^{2}$ aj. (89, 84 e $82 \%$, respectivamente) e baixo EP (5, 5 e 7\%, respectivamente) (Tabela 2). Nesse sentido, mais do que $80 \%$ da carga de $1 \mathrm{RM}$ podem ser determinados com as equações elaboradas, apresentando menos do que 7\% de erro.

Uma importante contribuição que o presente estudo traz é que os três exercícios foram explorados isoladamente para mulheres (supino horizontal, puxador costas e mesa flexora), resultando em três equações distintas. A maioria dos estudos que elaborou equações preditivas estudou somente o exercício supino horizontal. Nesse sentido, alguns estudos exploraram o supino horizontal isoladamente para mulheres ${ }^{9,16,17,19}$, em conjunto para mulheres e homens ${ }^{10}$, em conjunto com outros exercícios para mulhere ${ }^{20}$, ou ainda, em conjunto com outros exercícios para homens e mulheres ${ }^{18}$. Em contrapartida, poucos exploraram outros exercícios para mulheres isoladamente ${ }^{15}$ ou em conjunto com homens ${ }^{3,21}$.
Com relação aos valores de variância explicada, encontradas no presente estudo, podemos observar que, para o supino horizontal, este valor (89\%) foi igual ou superior ao observado em outros estudos realizados com o mesmo exercício ${ }^{16,17,19}$. Cummings e Finn ${ }^{19}$ avaliaram 57 mulheres não treinadas e encontraram que a relação entre $4-8$ repetições máximas e a carga utilizada apresentaram $\mathrm{R}^{2} a j$. de 0,89. Já Kim et al. ${ }^{16}$, utilizando a carga fixa de 15,9kg, avaliaram 16 estudantes de Medicina nas cadências de 30 e 60 repetições por minuto, encontrando $R^{2}$ aj. de 0,75 e 0,82 , respectivamente. Horvat et al. ${ }^{17}$ conduziram um estudo com 65 mulheres atletas de diferentes esportes, em que as equações foram elaboradas a partir do número máximo de repetições realizados em duas cargas fixas (25 e $31,8 \mathrm{~kg}$ ), em conjunto com a massa magra. Os valores de $\mathrm{R}^{2}$ aj. observados foram de 0,79 e 0,83 para as cargas de 25 e $31,8 \mathrm{~kg}$, respectivamente.

Para o exercício puxador costas, o valor de $\mathrm{R}^{2} \mathrm{aj}$. $(0,84)$ e EP $(5 \%)$, observados neste estudo, foram similares ao observado no estudo de Kuramoto e Payne $^{15}$, tanto para mulheres jovens (entre 20 e 30 anos) e de meia-idade (entre 40 e 50 anos) (R 2 aj. $=0,89 ; \mathrm{EP} \approx 5 \%$ ) como para mulheres mais velhas (entre 60 e 70 anos) ( $\mathrm{R}^{2} \mathrm{aj} .=0,81 ; \mathrm{EP} \approx 6 \%$ ). Por sua vez, no estudo de Johnson et al..$^{21}$, conduzido com mulheres universitárias treinadas, a relação entre número máximo de repetições realizados em $80 \%$ de 1RM só foram responsáveis por 19\% da variância explicada, com erro de $14 \%$. Do nosso conhecimento, nenhum estudo foi conduzido com mulheres, no exercício mesa flexora, impossibilitando que os valores de Rªj e EP sejam comparados.

Uma vantagem do presente estudo é o fato das equações terem sido elaboradas com grande amplitude de número de repetições, o que facilita a utilização prática destas equações. Alguns estudos observaram que uma intensidade específica de $1 \mathrm{RM}^{8,22}$ ou um número pré-determinado de repetições $^{18}$ são melhores preditores da carga de 1RM por apresentarem maiores valores de variância explicada. De forma similar com relação a restrição da intensidade ou número máximo de 
repetições, Johnson et al. ${ }^{21}$ utilizaram somente o número máximo de repetições realizados em $80 \%$ de 1RM, enquanto Cummings e Finn ${ }^{19}$ utilizaram somente 4-8 repetições máximas. A utilização das equações com porcentagens específicas de 1RM é inviável, dado que para encontrarmos uma intensidade relativa de $1 \mathrm{RM}$ é preciso, primeiramente, encontrar o valor de 1RM e, portanto, inutiliza o uso das equações para achar o valor de 1RM.

A mesma inviabilidade pode ser encontrada para a utilização de uma faixa pré-determinada de repetições. É comum observarmos que uma determinada faixa do número máximo de repetições tem sido relacionada com uma intensidade específica de 1RM. Porém, a literatura tem demonstrado, ainda que de maneira não consensual, que o número máximo de repetições que pode ser realizado em uma mesma intensidade, pode variar entre os gêneros ${ }^{2,23}$ e exercícios ${ }^{2,424}$. Além disso, quando um estudo apresenta o número mínimo e máximo de repetições executadas pelos participantes, é possível observar uma grande variação para o mesmo exercício e intensidade. Para o supino horizontal em $80 \%$ de 1RM, por exemplo, Ferreira et al..$^{25}$ demonstraram variação entre 8 e 19 repetições máximas, enquanto no estudo de Chagas et al..$^{24}$ a variação ficou entre 3 e 5 repetições máximas, variação essa que também foi observada no presente estudo (Tabela 1), demonstrando que a variação do número máximo de repetições ocorre tanto entre os indivíduos de um mesmo estudo, quanto entre os estudos. Braith et al. ${ }^{3}$, por exemplo, avaliaram 25 mulheres e 33 homens no exercício cadeira extensora, objetivando predizer a carga de 1RM a partir da carga utilizada no teste de 7-10 repetições máximas, pré e após um período de treinamento de 18 semanas. Os avaliados autosselecionaram cargas superiores no teste realizado após o treinamento (aproximadamente $80 \%$ de 1RM) comparadas àquelas selecionadas antes do treinamento (aproximadamente $70 \%$ de 1RM) ( $<<0,01)$. Consequentemente, foi elaborada uma equação no pré-treinamento e outra equação no pós-treinamento, haja vista que a equação pré-treinamento superestimou a carga de $1 \mathrm{RM}$ no pós-treinamento.

Outras variáveis que foram estudadas pela possibilidade de serem relacionadas com a carga de 1RM e, portanto, possíveis de serem incluídas na predição de 1RM para mulheres, foram as variáveis antropométricas, tanto isoladamente ${ }^{9}$ como em conjunto com o número máximo de repetições realizado em cargas submáximas ${ }^{16,17,19,21}$. As variáveis antropométricas apresentam correlação com a carga de 1RM, porém a sua utilização como preditoras de 1RM não são consensuais. Scanlan et al. ${ }^{9}$ observaram que as variáveis antropométricas não podem ser utilizadas para predizer a carga de 1RM no supino horizontal para mulheres universitárias não treinadas. Esses achados foram corroborados por Kim et al. ${ }^{16}$ ao avaliarem estudantes universitárias de Medicina para o mesmo exercício, e por Reynolds et al. ${ }^{18}$ para mulheres e homens nos exercícios supino máquina e leg-press. Por outro lado, estudos conduzidos com mulheres ${ }^{19,21}$ observaram aumento da variância explicada com a inclusão das variáveis antropométricas. Ainda que seja possível a inclusão dessas variáveis para aumentar a predição da carga de 1RM, a utilização das mesmas aumentaria o tempo gasto para as avaliações, contrapondo o objetivo das equações preditivas que é a redução do tempo com esses procedimentos.

A limitação do presente estudo é a ausência do processo de validação cruzada. Recomenda-se que estudos futuros façam a validação dessas equações para diferentes populações, considerando: (a) diferentes períodos de experiência com o treinamento de força; (b) diferentes objetivos das participantes com relação aos protocolos de treinamento de força; (c) sensibilidade para monitoramento do progresso do treinamento de força.

\section{CONCLUSÕES}

Ao analisar em conjunto os valores de $R^{2} a j$., demonstramos a viabilidade em estimar a carga correspondente a 1RM a partir das equações geradas com um erro reduzido (5-7\%), para mulheres familiarizadas com treinamento de força nos exercícios supino horizontal, puxador costas e mesa flexora através do número máximo de repetições em cargas submáximas. Entretanto, é recomendado que as cargas submáximas ou preditivas resultem em uma variação do número de repetições máximas entre 1 a 21 para o supino horizontal, 2 a 20 para o puxador costas e de 1 a 14 para mesa flexora.

Sendo assim, o uso das equações demonstrou ser um método eficiente para estimar a carga máxima, principalmente, pela redução do tempo gasto durante os procedimentos dos testes comparado ao método tradicional. Essa facilidade permite que os profissionais possam avaliar os alunos com maior periodicidade, possibilitando parâmetros mais eficientes para a estimativa da força máxima. Para cada exercício, o profissional gastará em média 5 minutos para aplicação do teste enquanto que, no método tradicional, ele gastaria aproximadamente entre 20 e 30 minutos. 


\section{Agradecimentos}

Nós gostaríamos de agradecer ao Programa Institucional de iniciação Científica PIVIC/ Mackenzie, que possibilitou a realização do presente estudo.

\section{REFERÊNCIAS BIBLIOGRÁFICAS}

1. American College of Sports Medicine (ACSM). Position Stand: The recommended quantity and quality of exercise for developing and maintaining cardiorespiratory and muscular fitness, and flexibility in healthy adults. Med Sci Sports Exerc 1998;30(6):975-91.

2. Hoeger WWK, Hopkins DR, Barette SL, Hale DF. Relationship between repetitions and selected percentages of one repetition maximum: a comparison between untrained and trained males and females. J App Sports Sci Res 1990;4(2):47-54.

3. Braith RW, Graves JE, Leggett SH, Pollock ML. Effect of training on the relationship between maximal and submaximal strength. Med Sci Sports Exerc 1993;25(1):132-8.

4. Shimano T, Kraemer WJ, Spiering BA, Volek JS, Hatfield DL, Silvestre R, et al. Relationship between the number of repetitions and selected percentages of one repetition maximum in free weight exercises in trained and untrained men. J Strength Cond Res 2006;20(4):819-23.

5. Fleck SJ, Kraemer WJ. Designing Resistance Training Programs 3rd ed. Champaign: Human Kinetics; 2004.

6. Ware JS, Clemens CT, Mayhew JL, Johnston TJ. Muscular endurance repetitions to predict bench press and squat strength in college football players. J Strength Cond Res 1995;9(2):99-103.

7. Mayhew JL, Ware JS, Bemben MG, Wilt B, Ward TE, Farris B, et al. The NFL-225 test as a measure of bench press strength in college football players. J Strength Cond Res 1999;13(2):130-4.

8. Kravitz L, Akalan C, Nowicki K, Kinzey SJ. Prediction of 1 repetition maximum in high-school power lifters. J Strength Cond Res 2003;17(1):167-72.

9. Scanlan JM, Ballmann KL, Mayhew JL, Lantz CD. Anthropometric dimensions to predict 1-RM bench press in untrained females. J Sports Med Phys Fitness 1999; 39(1):54-60.

10. Mayhew JL, Ball TE, Arnold MD, Bowen JC. Relative muscular endurance performance a predictor of bench press strength in college men and woman. J App Sport Sci Res 1992;6(4):200-6.

11. Desgorces FD, Berthelot G, Dietrich G, Testa MSA. Local muscular endurance and prediction of 1 repetitions maximum for bench in 4 athletic populations. J Strength Cond Res 2010;24(2):394-400.

12. Mayhew JL, Johnson BD, Lamonte MJ, Lauber D, Kemmler W. Accuracy of prediction equations for determining one repetition maximum bench press in women before and after resistance training. J Strength Cond Res 2008;22(5):1570-7.

13. Miller AEJ, Macdougall JD, Tarnopolsky MA, Sale DG. Gender differences in strength and muscle fibre characteristics. Eur J Appl Physiol 1993;66(3):254-62.
14. Brown LE, Weir JP. ASEP Procedures Recommendation I: Accurate Assessment of Muscular Strength and Power. J Exerc Physiol 2001;4(3):1- 21.

15. Kuramoto AK, Payne VG. Predicting muscular strength in women: a preliminary study. Res Q Exerc Sport 1995;66(2):168-72.

16. Kim PS, Mayhew JL, Peterson DF. A modified YMCA bench press test as a predictor of 1 repetition maximum bench press strength. J Strength Cond Res 2002;16(3):440-5.

17. Horvat M, Ramsey V, Franklin C, Gavin C, Palumbo T, Glass LA. A method for predicting maximal strength in collegiate women athletes J Strength Cond Res 2003;17(2):324-8.

18. Reynolds JM, Gordon TJ, Robergs RA. Prediction of one repetition maximum strength from multiple repetition maximum testing and anthropometry. J Strength Cond Res 2006;20(3):584-92.

19. Cummings B, Finn KJ. Estimation of a one repetition maximun bench press for untrained women. J Strength Cond Res 1998;12(4):262-265.

20. Kemmler WK, Lauber D, Wassermann A, Mayhew JL. Predicting maximal strength in trained postmenopausal woman. J Strength Cond Res 2006;20(4):838-42.

21. Johnson D, Lynch J, Nash K, Cygan J, Mayhew JL. Relationship of lat-pull repetitions and pull-ups to maximal lat-pull and pull-up strength in men and women. J Strength Cond Res 2009;23(3):1022-8.

22. Morales J, Sobonya S. Use of submaximal repetitions tests for predicting 1-RM strength in class athtletes. J Strength Cond Res 1996;10(3):186-9.

23. Salvador EP, Cyrino ES, Gurjão ALD, Dias RMR, Nakamura FY, Oliveira AR. Comparação entre o desempenho motor de homens e mulheres em séries múltiplas de exercicios com pesos. Rev Bras Med Esporte 2005;11(5):257-61.

24. Chagas MH, Barbosa JRM, Lima FV. Comparação do número máximo de repetições realizadas a 40 e $80 \%$ de uma repetição máxima em dois diferentes exercícios na musculação entre os gêneros masculino e feminino. Rev Bras Educ Fis Esporte 2005;19(1):5-12.

25. Ferreira S, Marins JCB, Silva LC, Lunz W, Pimentel GGA, Migliorini EM. Determinação de perfil de repetições máximas no exercício de extensão de pernas e supino reto com diferentes percentuais de força. Rev Educ Fis 2006;17(2):149-59.

\section{Endereço para correspondência}

Ursula Ferreira Julio

Departamento de Esporte - Escola de Educação

Física e Esporte

Universidade de São Paulo

Av. Prof. Mello de Morais, 65, Butantã, 05508-900 - São Paulo, SP, Brasil

E-mail: ursulajulio@usp.br 